\title{
Low prevalence of Pneumocystis firovecif colonization in healthy population: New controversy
}

Objectives: Pneumocystis jirovecii colonization in general population has been poorly studied. Available data indicates high prevalence of $P$. jirovecii colonization among general population. These reports limited to some geographical regions. Some paradoxical reports indicating lower prevalence of $P$. jirovecii infection or colonization especially in subtropical regions. The aim of this study was to investigate the prevalence of $P$. jirovecii colonization in healthy population in a hot and dry weather in south of Iran.

Methods: Oropharyngeal wash samples were collected from 226 healthy people referring to four screening centers and occupational health services during the four seasons of the 2015 . Each participant passes a clinical-epidemiologic examination; they did not have any clinical signs of Pneumocystis pneumonia (PCP) or other diseases at the time of sampling or during the last six mounts previous the study .P. jirovecii DNA was detected in the samples using two nested-PCR methods targeting different targets; 18SrDNA and mtLSUrRNA of the organism.

\section{Sources:}

Khodadadi $\mathrm{H}$, Mirhendi $\mathrm{H}$, Mohebali $\mathrm{M}$, Kordbacheh $\mathrm{P}$, Zarrinfar H, Makimura K. (2013) Pneumocystis jirovecii Colonization in Non-HIV-Infected Patients Based on NestedPCR Detection in Broncho alveolar Lavage Samples. Iranian journal of public health, 42(3):298-305.

Morris A, Wei K, Afshar K, Huang L (2008). Epidemiology and Clinical Significance of Pneumocystis Colonization. J Infect Dis, 197:10 -7.
Results: 127 (56.19\%) and 99 (43.80\%) of participants were male and female respectively. The mean age of them was 34 years. $P$. jirovecii DNA was detected in 7 (3.09\%) out of 226 OPW samples by amplify both genes of the organism. There was no correlation between colonization and participants demographic parameter or seasonal variations $(p<0.05)$.

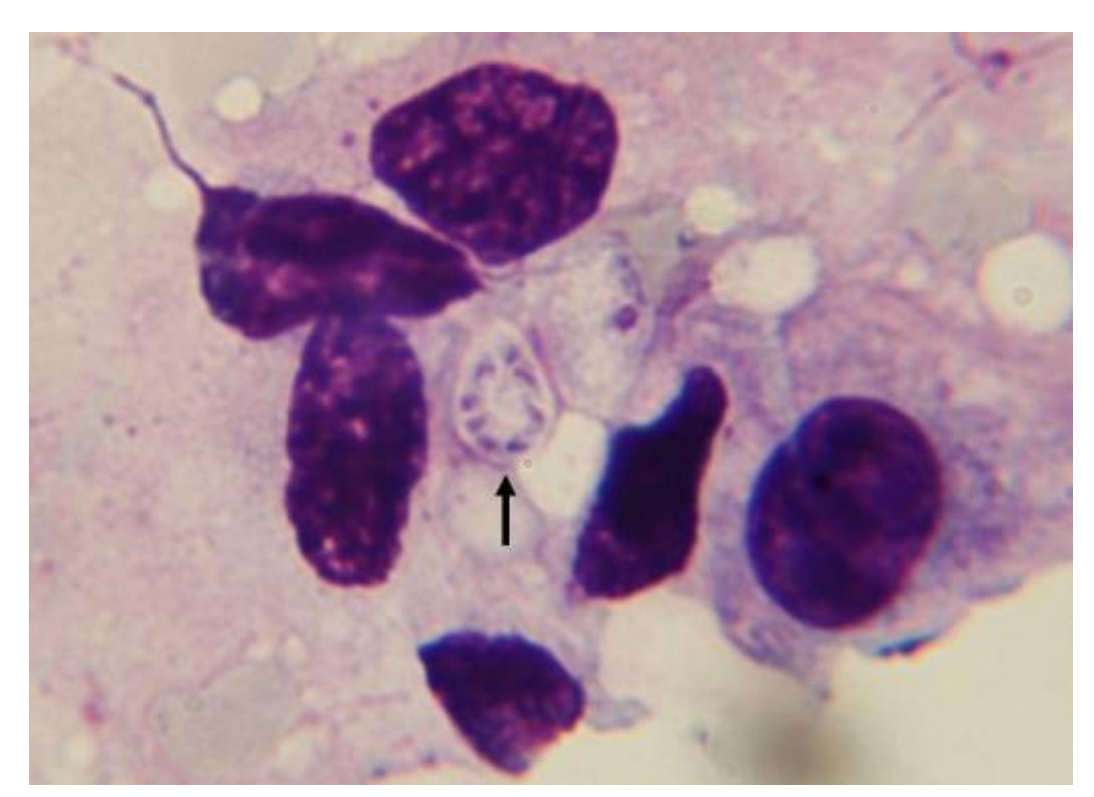

Conclusions: The low prevalence of Pneumocystis colonization in this study is in agreement with previous studies indicating lower detection of $P$. jirovecii in patients without risk factors for pneumocystosis in this region. However there is an obvious difference between our findings and some previous reports from other part of the world. This finding indicates necessity of further multicenter analyses on $P$. jirovecii colonization and considering a prevalence-based data for epidemiologic studies in each region. Attention to different social and geographical conditions may change our aspect about Pneumocystis epidemiology especially on its natural reservoirs and routes of transmission. 